\title{
Correction to: Dominant and global dimension of blocks of quantised Schur algebras
}

\author{
Ming Fang ${ }^{1,2} \cdot$ Wei Hu$^{3} \cdot$ Steffen Koenig ${ }^{4}$ \\ Published online: 22 August 2021 \\ (c) Springer-Verlag GmbH Germany, part of Springer Nature 2021
}

\section{Correction to: Mathematische Zeitschrift https://doi.org/10.1007/s00209-021-02792-w}

Unfortunately, during production of this article the typsetter working for Springer production introduced various mistakes, failed to correct some of these after having received the authors' comments on galley proofs and introduced new mistakes while correcting other mistakes. None of these mistakes changes the mathematical contents, but carrying out these corrections improves readability of the article and is fair to authors and readers.

Corrections missed out by Springer production, in the form communicated to the Springer production team are as follows:

page 4, Proposition 2.2 Müller B [25] $\rightarrow$ Proposition 2.2 (Müller [25]), i.e. to be done: put parentheses and remove the letter $\mathrm{B}$.

page 4, first sentence of Sect. 2.2 Hecke algebras and Schur algebras "... and q a nonzero element": nonzero should be non-zero (this should be unified throughout the whole article, it seems to be unified everywhere else already).

The original article can be found online at https://doi.org/10.1007/s00209-021-02792-w.

Steffen Koenig

skoenig@mathematik.uni-stuttgart.de

Ming Fang

fming@amss.ac.cn

Wei Hu

huwei@bnu.edu.cn

1 HLM, HCMS, Academy of Mathematics and Systems Science, Chinese Academy of Sciences, Beijing 100190, People's Republic of China

2 School of Mathematical Sciences, University of Chinese Academy of Sciences, Beijing 100049, People's Republic of China

3 School of Mathematical Sciences, Laboratory of Mathematics and Complex Systems, MOE, Beijing Normal University, Beijing 100875, People's Republic of China

4 Institute of Algebra and Number Theory, University of Stuttgart, Pfaffenwaldring 57, 70569 Stuttgart, Germany 
page 7, Theorem 3.2 Fang et al. [11] $\rightarrow$ Theorem 3.2 (Fang et al. [11]), i.e. again put parentheses

pages 9 and 10: Here, remark (3) looks like it stops after one paragraph, which is not correct. It runs until the very end of the article (ending with "is not almost $\nu$ stable"). It had been like that in the manuscript and had been changed by production. We had already asked to revert to the correct way of arranging these remarks, which has not happened.

Acknowledgements

National Key R\&D Program of China $\rightarrow$ R\&D appearing here is html code, but it should of course be typeset in latex to be read as R\&D. Currently, it looks really strange.

According to the Springer production team, these mistakes are corrected in the version of the article appearing online at the time this correction appears there.

The original article has been corrected.

Publisher's Note Springer Nature remains neutral with regard to jurisdictional claims in published maps and institutional affiliations. 